\title{
Error-prone DnaE2 Balances the Genome Mutation Rates in Myxococcus xanthus DK1622
}

\author{
Ran Peng, Jiang-he Chen, Wan-wan Feng, Zheng Zhang, Jun Yin, Ze-shuo Li and \\ Yue-zhong $L i$ *
}

State Key Laboratory of Microbial Technology, School of Life Science, Shandong University, Jinan, China

$d n a E$ is an alpha subunit of the tripartite protein complex of DNA polymerase III that is responsible for the replication of bacterial genome. The dnaE gene is often duplicated in many bacteria, and the duplicated $d n a E$ gene was reported dispensable for cell survivals and error-prone in DNA replication in a mystery. In this study, we found that all sequenced myxobacterial genomes possessed two dnaE genes. The duplicate dnaE genes were both highly conserved but evolved divergently, suggesting their importance in myxobacteria. Using Myxococcus xanthus DK1622 as a model, we confirmed that dnaE1 (MXAN_5844) was essential for cell survival, while dnaE2 (MXAN_3982) was dispensable and encoded an error-prone enzyme for replication. The deletion of dnaE2

OPEN ACCESS

Edited by:

Feng Gao,

Tianjin University, China

Reviewed by:

Camilo E. Khatchikian,

University of Texas at El Paso, USA

Hao Wu,

University of Gothenburg, Sweden

*Correspondence: Yue-Zhong Li lilab@sdu.edu.cn

Specialty section:

This article was submitted to

Evolutionary and Genomic

Microbiology,

a section of the journal

Frontiers in Microbiology

Received: 05 November 2016 Accepted: 17 January 2017 Published: 01 February 2017

Citation:

Peng R, Chen J-h, Feng W-W, Zhang Z, Yin J, Li Z-s and Li Y-Z

(2017) Error-prone DnaE2 Balances the Genome Mutation Rates in Myxococcus xanthus DK1622.

Front. Microbiol. 8:122.

doi: 10.3389/fmicb.2017.00122 had small effects on cellular growth and social motility, but significantly decreased the development and sporulation abilities, which could be recovered by the complementation of $d n a E 2$. The expression of $d n a E 1$ was always greatly higher than that of $d n a E 2$ in either the growth or developmental stage. However, overexpression of dnaE2 could not make dnaE1 deletable, probably due to their protein structural and functional divergences. The dnaE2 overexpression not only improved the growth, development and sporulation abilities, but also raised the genome mutation rate of $M$. xanthus. We argued that the low-expressed error-prone DnaE2 played as a balancer for the genome mutation rates, ensuring low mutation rates for cell adaptation in new environments but avoiding damages from high mutation rates to cells.

Keywords: dnaE2 gene, dispensable, error-prone, chromosome replication, growth, development and sporulation, Myxococcus xanthus

\section{INTRODUCTION}

DNA polymerase III is an enzyme complex responsible for prokaryotic genome replication (Kelman and O'donnell, 1995). The holoenzyme consists of a DNA polymerase (polymerase III $\alpha$-subunit, PolIII $\alpha$ ), a processivity factor $\beta$-clamp and a clamp loader protein (McHenry, 2011a,b; Robinson et al., 2012). There are two types of PolIII $\alpha$ : dnaE and PolC, both of which belong to the C-family of DNA polymerase (Ito and Braithwaite, 1991). While PolC exists in low-GC Gram-positive bacteria such as Bacillus subtilis, dnaE is universally distributed in different kinds of bacterial cells (Evans et al., 2008). The dnaE gene is essential in bacteria, and its functions have been well elucidated in some bacteria, such as Escherichia coli (Lamers et al., 2006). In addition to the essential $d n a E$ gene, many bacterial species have evolved a second $d n a E$ copy, usually named as $d n a E 2$. For example, duplicated $d n a E$ genes are often existed in those bacteria possessing large-sized genomes with high $\mathrm{G}+\mathrm{C}$ contents (Zhao et al., 2007). dnaE2 normally exists in a three-gene 
operon in Proteobacteria, accompanying with imuA and $i m u B$, and the operon is regulated by LexA2, a possible transcriptional regulator associated with DNA damage response (Abella et al., 2004). Previous genetic studies indicated that dnaE2 was nonessential for the chromosomal DNA replication in bacterial cells (Boshoff et al., 2003; Abella et al., 2004; Warner et al., 2010; Tsai et al., 2012). In Streptomyces, DnaE2 was reported to be an error-prone enzyme, associating with the DNA damageinducible translesion DNA synthesis (Tsai et al., 2012). In Mycobacterium tuberculosis, the error-prone DnaE2 was proved in vitro to act as a primary mediator for cell survival through inducing mutagenesis and thus contributing directly to the emergence of drug resistance (Boshoff et al., 2003). However, this function is not in prevalence. For example, researches in Pseudomonas and Streptomyces indicated that dnaE2 was not required for replication, end patching, or ultraviolet resistance and mutagenesis (Koorits et al., 2007; Tsai et al., 2012). The role of the replicated DnaE2 remains mostly unclear yet.

Myxobacteria are phylogenetically located in the delta division of the Proteobacteria (Shimkets et al., 2006). The bacteria are widely distributed in various environmental conditions, playing as micropredators by feeding on other microbial cells or macromolecules (Reichenbach, 1999; Jiang et al., 2010; Brinkhoff et al., 2012; Li et al., 2012; Zhou et al., 2014). Myxobacteria are characterized among the Prokaryotes by their complex multicellular social behaviors: cells locomote on solid surfaces in swarms to collaboratively prey on other microbial cells and, when food is scarce, aggregate to develop multicellular fruiting bodies, inside which differentiate metabolically quiescent myxospores (Shimkets, 1990; Kaiser and Losick, 1993; Dworkin, 1996; Shimkets et al., 2006). In this study, we bioinformatically analyzed those sequenced myxobacteria, and found that each of the myxobacterial genomes possessed two $d n a E$ genes. The duplicate myxobacterial $d n a E$ genes were both highly conserved but evolved divergently. Using Myxococcus xanthus DK1622 as a model, we confirmed that one $d n a E$ gene was essential for cell survival, while the other was dispensable and error-prone. We evaluated functions of the $d n a E$ genes and assayed their expressions in vegetative growth stage and developmental stage. It is known that there are two processes involving the genome replication in $M$. xanthus, one in the growth stage and the other in the early development stage (Tzeng and Singer, 2005). We found that the non-essential dnaE gene played functions in both of the two replication processes in M. xanthus DK1622. We suggested that the error-prone DnaE2 played as a balancer for the genome mutation rates via expression regulation, thus ensuring low mutation rates for the adaptation in new environments and avoiding high mutation rates to damage cells.

\section{RESULTS}

\section{dnaE1 Is Essential While dnaE2 Is Dispensable and Encodes An Error-prone DNA Polymerase in $M$. xanthus}

Myxobacterial genomes are normally larger than nine $\mathrm{Mbp}$ in size, but with some exceptions of approximate
five-Mbp-genomes in Anaeromyxobacter strains. The $\mathrm{G}+\mathrm{C}$ contents of the sequenced myxobacterial genomes ranged from $67.4 \%$ in Enhygromyxa salina DSM 15201 to $74.9 \%$ in Anaeromyxobacter dehalogenans 2CP-C. All the sequenced myxobacterial genomes, no matter in large size or small size, contained two dnaE genes (Table S1). Phylogenetic analysis indicated that the two protein sequences encoded by the duplicate myxobacterial $d n a E$ genes were clustered separately with determined DnaE1 and DnaE2 of other bacteria, forming the DnaE1 and DnaE2 groups (Figure S1). The single DnaE gene of E. coli was in the DnaE1 group, clearly distant from the DnaE2 group; and the two DnaE-group trees had highly similar topologies. Consistent with the previous results (Timinskas et al., 2014; Wu et al., 2014), the emergence of myxobacterial dnaE2 was derived from an early duplication event of the primordial $d n a E$. High conservation but great divergence suggested that the duplicate DnaE polymerases were both important for myxobacterial cells, but functioned divergently.

M. xanthus DK1622 is the model strain of myxobacteria. In the genome of DK1622, two genes, i.e., MXAN_3982 and MXAN_5844, were predicted to be dnaE, encoding for the alpha subunit of DNA polymerase III (Goldman et al., 2006). The proteins encoded by the two $d n a E$ genes contain 1185 and 1013 amino acids (MXAN_5844 and MXAN_3982), respectively. These two proteins were phylogenetically distant, having $48 \%$ similarity and 30\% identity of their amino acid sequences. According to their phylogenetic locations (Figure S1), the MXAN_3982 gene was designated as dnaE2, and the MXAN_5844 gene was dnaE1.

To evaluate their essentiality, we made deletion mutations of the dnaE1 and dnaE2 gene in M. xanthus DK1622, respectively. While dnaE2 was deletable, producing the mutant of YL1601, the dnaE1 gene could not be deleted, which was further confirmed by failed attempts to delete the dnaE1 gene in a dnaE2overexpressing mutant (see below). Compared with that of the wild-type strain DK1622, the OD values of the dnaE2 deletion mutant YL1601 were smaller than the wild type at different time points during the exponential growth stage in CTT growth medium (two-way ANOVA, $P<0.01$; Figure 1A). To confirm the function of $d n a E 2$ in growth, we further constructed a mutant by inserting the dnaE2 gene following its own promoter at the attB site in the YL1601 genome, forming the YL1606 mutant. As expected, the complementary strain recovered the delayed growth in YL1601, showing almost the same growth curve as the wild type strain DK1622 (Figure 1A).

To confirm whether the DnaE2 protein was an error-prone enzyme, we constructed two more mutants by deleting two DNArepair genes of mutL (MXAN_4026) and mutS (MXAN_3897) from DK1622, respectively, producing the mutants of YL1602 and YL1603. The wild type strain DK1622 and the three mutants of YL1601, YL1602 and YL1603 were cultivated on CTT growth medium supplemented with nalidixic acid $(40 \mu \mathrm{g} / \mathrm{ml})$ to evaluate their production abilities of resistant mutations. It is known that the inhibition of nalidixic acid on DNA repair can be reversed by the MutL and MutS enzymes of the DNA repairing system in $E$. coli cells, thus the deletion mutant of mutL or mutS will produce more genome mutations than the wild type strain during 

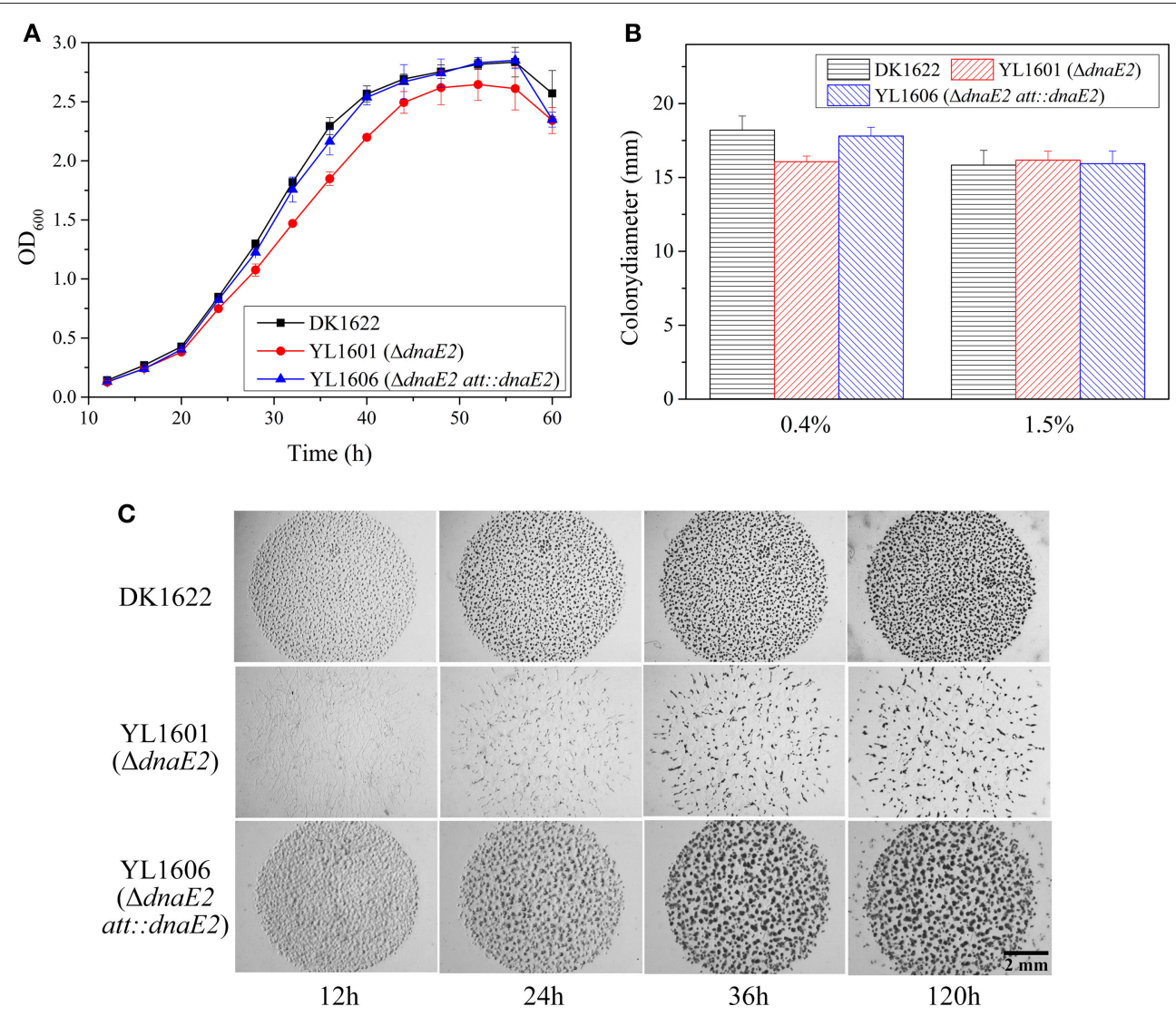

FIGURE 1 | Comparison of phenotypic characteristics of the dnaE2 mutant (YL1601), the dnaE2-complementary mutant (YL1606) and the wild type strain DK1622. (A) Growth curves of YL1601, YL1606, and DK1622 in CTT liquid medium. OD600 values were measured every 4 h until 60 h of incubation. The data were analyzed using two-way ANOVA. (B) Cellular swarming abilities on 0.4 and $1.5 \%$ agar CTT plate. (C) Aggregation and formation abilities of fruiting bodies on TPM development plate. The error bars represent the standard deviation of three independent experiments.

the chromosome replication process (Sniegowski et al., 1997). In M. xanthus, the mutation rate of DK1622 was approximately 3.69 $\times 10^{-8}$ per nucleotide in the presence of nalidixic acid; whereas the YL1602 and YL1603 mutants produced mutations of 5.41 $\times 10^{-7}$ and $1.77 \times 10^{-6}$, respectively (Table 1 ). The mutation rates of the mutL and mutS mutants were both significantly higher than that of DK1622 ( $t$-test, $P<0.01)$. This result was consistent with that reported in E. coli (Sniegowski et al., 1997), suggesting the method was also reliable in $M$. xanthus for the evaluation of mutation rates. As expected, the deletion of $d n a E 2$ made the mutant, possessing only $d n a E 1$, to have nearly 10 times lower of mutation rate than that of the wild type strain DK1622, which harbors both dnaE1 and dnaE2 (Table 1). This result confirmed the error-prone characteristics of the DnaE2 protein in M. xanthus cells.

\section{Effects of the Deletion of dnaE2 on M. xanthus Social Behaviors}

The above results indicated that $d n a E 1$ was essential for the survival of $M$. xanthus cells, while the error-prone DnaE2 gene was dispensable for cell survival. dnaE2 also involved in cellular growth, but in weak effects and low fidelity. We further assayed effects of the deletion of dnaE2 in the swarming and developmental processes in $M$. xanthus. Similar to the growth change, the swarming ability of the YL1601 mutant was weakly decreased on $0.4 \%$ CTT plate, comparing with that of DK1622 ( $t$-test, $P=0.051$ ), while the motility on $1.5 \%$ CTT plate showed no difference between DK1622 and YL1601 (Figure 1B).

When cultivated on the TPM developmental medium, cells of the dnaE2 deletion mutant aggregated irregularly (Figure 1C). From the phenotypes of the mutant on TPM, the dnaE2 gene probably played functions in the early stage of the aggregation in the development process. The sporulation ability of the dnaE2 mutant was decreased to approximately $70 \%$ of that of DK1622 after 5 days of incubation on TPM medium $\left(1.49 \times 10^{6} \pm 9.61\right.$ $\times 10^{4}$ vs. $2.14 \times 10^{6} \pm 1.70 \times 10^{5}$; Table 2). If the dnaE2 gene was complemented into the dnaE2 deletion mutant (YL1606), the irregular aggregation phenotype on TPM developmental medium was completely recovered (Figure 1C). This result suggested that the DnaE2 protein also played functions in the early development stage of $M$. xanthus DK1622 cells. Seemly, the function of the dnaE2 gene was more obvious in the development stage than its function in the growth stage. 
TABLE 1 | Mutation rates of M. xanthus DK1622 and mutants grown on CTT plates supplemented with nalidixic acid.

\begin{tabular}{|c|c|c|c|c|}
\hline Strains & Cell num. & Average count & Mutation rate & St.dev \\
\hline DK1622 & $5.0 \times 10^{9}$ & 185 & $3.69 \times 10^{-8}$ & $2.05 \times 10^{-9}$ \\
\hline YL1602 (smutS) & $5.0 \times 10^{9}$ & 2707 & $5.41 \times 10^{-7}$ & $5.05 \times 10^{-8}$ \\
\hline YL1603 (smutL) & $5.0 \times 10^{9}$ & 8842 & $1.77 \times 10^{-6}$ & $2.24 \times 10^{-7}$ \\
\hline YL1601 ( $\Delta d n a E 2)$ & $5.0 \times 10^{9}$ & 24 & $4.87 \times 10^{-9}$ & $5.03 \times 10^{-10}$ \\
\hline YL1604 (DK1622 att::Kan) & $5.0 \times 10^{9}$ & 214 & $4.28 \times 10^{-8}$ & $2.05 \times 10^{-9}$ \\
\hline YL1605 (DK1622 att::dnaE2) & $5.0 \times 10^{9}$ & 682 & $1.36 \times 10^{-7}$ & $1.86 \times 10^{-8}$ \\
\hline
\end{tabular}

TABLE 2 | Sporulation abilities of M. xanthus DK1622 and YL1601 ( $\Delta$ dnaE2) cells grown on TPM plates treated with nalidixic acid for1 h at different time points.

\begin{tabular}{|c|c|c|c|c|}
\hline Time of addition & Spore production in DK1622 & Percentage of control\% & Spore production in YL1601 & Percentage of control\% \\
\hline Control & $2.14 \times 10^{6} \pm 1.70 \times 10^{5}$ & 100 & $1.49 \times 10^{6} \pm 9.61 \times 10^{4}$ & 100 \\
\hline $\mathrm{Oh}$ & $1.52 \times 10^{5} \pm 9.60 \times 10^{3}$ & 7.13 & $5.53 \times 10^{4} \pm 6.51 \times 10^{3}$ & 3.72 \\
\hline $6 \mathrm{~h}$ & $1.45 \times 10^{6} \pm 1.11 \times 10^{5}$ & 68.02 & $3.50 \times 10^{5} \pm 3.61 \times 10^{4}$ & 23.54 \\
\hline $12 \mathrm{~h}$ & $2.12 \times 10^{6} \pm 1.31 \times 10^{5}$ & 99.22 & $7.67 \times 10^{6} \pm 4.51 \times 10^{4}$ & 51.57 \\
\hline $18 \mathrm{~h}$ & $2.21 \times 10^{6} \pm 1.99 \times 10^{5}$ & 103.59 & $1.08 \times 10^{6} \pm 2.10 \times 10^{5}$ & 72.65 \\
\hline $24 \mathrm{~h}$ & $2.33 \times 10^{6} \pm 1.21 \times 10^{5}$ & 108.89 & $1.54 \times 10^{6} \pm 1.12 \times 10^{5}$ & 103.81 \\
\hline
\end{tabular}

The sporulation ability was assayed after 5 days of incubation on TPM.

\section{Functions of dnaE2 in the Chromosome Replication Progress in Development}

Tzeng and Singer found that there is a DNA replication process during the development of fruiting bodies and sporulation in M. xanthus DK1622 (Errington and Wake, 1991; Tzeng and Singer, 2005). Accordingly, there are two processes involving the chromosome replication during the lifecycle of $M$. xanthus cells: one in the growth stage and the other in the development stage. To determine DnaE2 function in development, we inoculated concentrated cells of DK1622 and YL1601 on the TPM plates, and exposed culture plates with nalidixic acid solution at the final concentration of $20 \mu \mathrm{g} / \mathrm{ml}$ for $1 \mathrm{~h}$ at different cultivation time points. After $120 \mathrm{~h}$ of incubation, the sporulation rates of the cultures were calculated and compared with that of their respective controls without the exposure of nalidixic acid. Consistent with the previous report (Tzeng et al., 2006), the developmental progress of DK1622 was arrested by nalidixic acid in the early stage of development. After $12 \mathrm{~h}$ of incubation, the nalidixic acid exposure had nearly no effect on the sporulation of M. xanthus DK1622 cells (Table 2). In contrast, the sporulation ability of the dnaE2 deletion mutant YL1601 was lower than that of DK1622 after the nalidixic acid exposure at each time point. The developmental arrest in YL1601 by the exposure of nalidixic acid was released after $24 \mathrm{~h}$, which was nearly two-times long of that in DK1622. This result indicated that the deletion of dnaE2 markedly prolonged the process of chromosome replication in development. Thus, together with the dnaE1 gene, the dnaE2 gene involved in the two processes of chromosome replication during the lifecycle of $M$. xanthus cells; its function in the development progress seemed to be more significant.

\section{dnaE1 Expression Is Greatly Higher than That of dnaE2}

There are two possible reasons for the differentiated functions of the dnaE1 and dnaE2 genes in the growth and developmental stages: structure differences and/or expression levels. We further assayed expression levels of the dnaE1 and dnaE2 genes under either the growth or the developmental conditions. Quantitative real-time PCR amplification showed that the expression levels of $d n a E 1$ were greatly higher than that of $d n a E 2$ at different time points under the CTT nutritional growth conditions (Figure 2A). For example, after $12 \mathrm{~h}$ of incubation in CTT liquid medium, the expression of dnaE1 was more than 10 times of that of $d n a E 2$. With the increase of incubation time, the expressed products of dnaE1 were gradually decreased and reached to less than onetenth $(8.7 \%)$ of the 12 -h-level at $48 \mathrm{~h}$ of incubation. Similarly, the expressions of $d n a E 2$ were also gradually decreased and were always significantly lower than that of $d n a E 1$ at each time point ( $t$-test, $P<0.01)$.

The expressions of the two dnaE-genes were in similar patterns on the TPM development plate as they were in the growth conditions (Figure 2B). In contrast, the expressions of dnaE2 in the developmental conditions were even lower than that in the growth conditions. After the deletion of dnaE2, no expression of dnaE2 was detectable in the mutant, and the expression of the $d n a E 1$ gene was lower than that of the wild type strain at $6 \mathrm{~h}$ of incubation on the TPM plate (71.5\%; Figure 2C). The results suggested that the deficiencies in the fruiting body formation and sporulation in the YL1601 mutant were probably resulted not only from the loss of $d n a E 2$, but also from the decreased expression level of $d n a E 1$. 

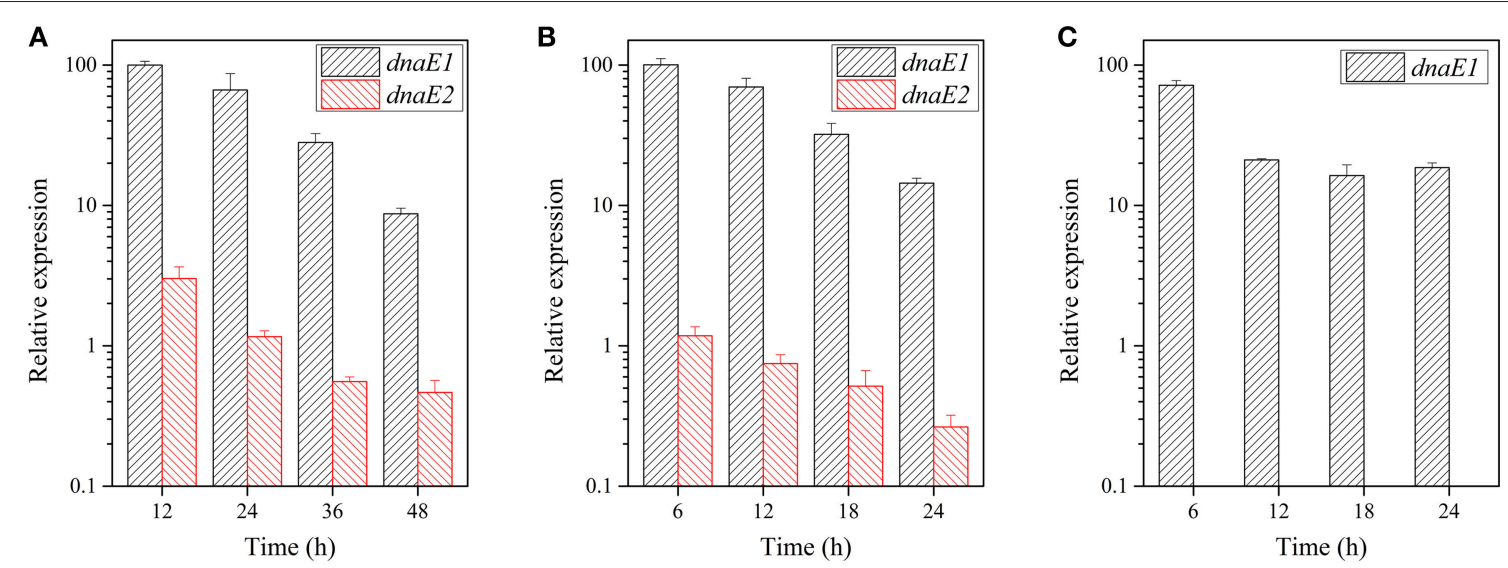

FIGURE 2 | Expression levels of the dnaE1 and dnaE2 genes at different time points under the nutritional and developmental conditions. (A) M. xanthus DK1622 cells grown in the CTT growth medium. (B) The DK1622 strain grown on the TPM development plate. (C). The YL1601 mutant cells grown on TPM plate. The expression of the $d n a E 1$ gene in CTT at $12 \mathrm{~h}$ of incubation was set as 100, and the others were the relative expressions. The error bars represent the standard deviation of three independent experiments.

\section{Overexpression of dnaE2 Improves Growth, Development, and Mutation Rate}

To further investigate effects of the dnaE2 expression, we introduced a second copy of the gene into the attB site of DK1622 chromosome by the Mx8 integrase in pSWU19 (Wu and Kaiser, 1995), producing the YL1605 mutant (containing a local dnaE2 and an introduced dnaE2). The introduced dnaE2 gene was designed to follow the pilA promoter, a high efficient promoter in $M$. xanthus cells. Meanwhile, a mutant containing the empty pSWU19 plasmid was also constructed into the att $B$ site of DK1622 chromosome as control (the mutant YL1604). The total expression levels of the two dnaE2 genes were approximately 10 times higher than that of $d n a E 1$ in the YL1605 mutant under either the nutritional (Figure 3A; at $24 \mathrm{~h}$ of incubation in CTT; $t$-test, $P<0.01$ ) or the developmental (Figure 3B; at $6 \mathrm{~h}$ of incubation on TPM; $t$-test, $P<0.01)$ conditions. In contrast, the expressions of dnaE2 in YL1605 were approximately 500 times and 1500 times higher than that in YL1604 under the nutritional and the developmental conditions, respectively. These results suggested that the overexpression of $d n a E 2$ was mostly resulted from the introduced dnaE2 gene. When cultivated in CTT liquid medium, the OD values of the dnaE2 overexpression mutant YL1605 were higher than the control mutant YL1604 during the early exponential growth stage (Figure 3C). The growth differences were statistically significant (two-way ANOVA, $P<$ 0.01 ). This result indicated that while the deletion of dnaE2 led to weakly delayed growth, overexpression of the gene could weakly increase cellular growth.

Interestingly, the overexpression of dnaE2 made the YL1605 mutant to have nearly three times higher of mutation rate than that of YL1604 cultivated on CTT plates supplemented with high concentrations of nalidixic acid $\left(1.36 \times 10^{-7}\right.$ vs. $4.28 \times 10^{-8}$, referred to Table 1). The difference was statistically significant $(t$-test, $P<0.01)$. In order to verify whether the overexpression changed the functions of dnaE2 in the development progress, we exposed the TPM cultures of YL1605 and YL1604 mutants with nalidixic acid solution for $1 \mathrm{~h}$ at the same time points described above (Table 3). The results showed that the dnaE2overexpressing mutant also had higher sporulation ability than the control when cells were treated with nalidixic acid in the early stage of development (before $12 \mathrm{~h}$ of incubation). Thus, overexpression of the dnaE2 gene was not only able to increase the genome mutation rate, but also speed up the development progress. However, compared with effects of the dnaE2 deletion, overexpression of $d n a E 2$ produced rather weak effects. The dnaE1 gene still played major roles in the chromosome replication.

\section{Overexpression of dnaE2 Does Not Take Place of dnaE1}

Imbalanced effects of the deletion and overexpression of $d n a E 2$ suggested that the structural differences of the two DnaE proteins played more important roles for function than their expressions. Structural differences between DnaE2 and DnaE1 have been thoroughly investigated (Warner et al., 2010; McHenry, 2011a,b; Timinskas et al., 2014). To determine whether the essentiality of dnaE1 was due to high expressions in DK1622, we retried to knock out the dnaE1 gene in the dnaE2-overexpressing mutant (YL1605) using the pBJ113Cm-5844 plasmid but failed. This result indicated that dnaE2 could not replace dnaE1 even if the dnaE2 gene was overexpressed, which confirmed that the non-fungibility of DnaE1 was due to the structural differences between DnaE1 and DnaE2. We modeled three-dimensional structures of DK1622 DnaE1 and DnaE2 (Figure 4A), using the threading approach (Yang et al., 2015). The estimated TM-scores are $0.84 \pm 0.08$ and $0.87 \pm 0.07$ for the modeled structures of DnaE1 and DnaE2, respectively. In general, the modeled protein structure with a TM-score $>0.5$ is acceptable with the correct topology (Yang et al., 2015). The DnaE1 and DnaE2 proteins of $M$. xanthus DK1622 had highly similar three-dimensional 
structures, even though their sequences were highly different. The active sites and some of the DNA binding surfaces were also highly conserved in the DnaE1 and DnaE2 polymerases. In addition, the two DnaE proteins were also considerably conserved of their surface amino acid positions (Figure 4A, right panels). However, the DnaE1 polymerase contained five domains, including PHP, Pol3, HhH, OB, and CTD domains, while DnaE2 had four domains, lacking the CTD domain (Figure 4A, left
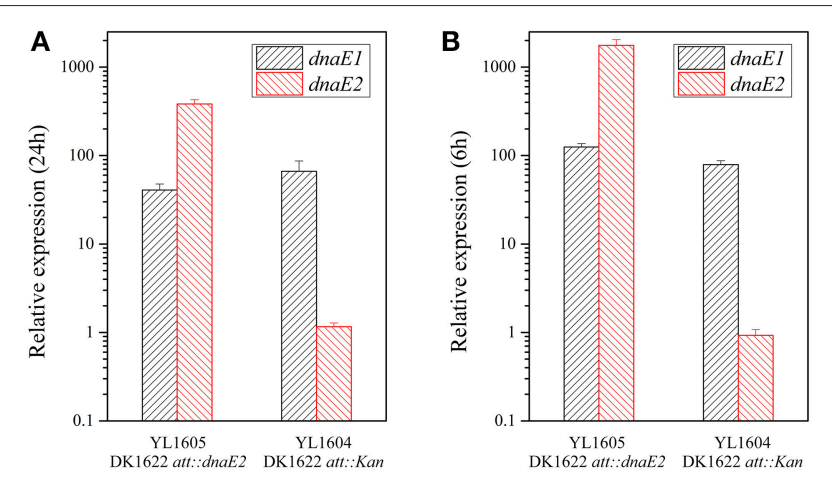

C

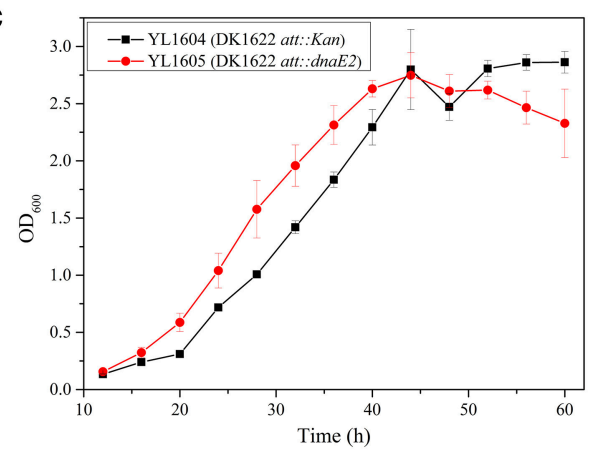

FIGURE 3 | Overexpression of the dnaE2 gene in $M$. xanthus cells and cellular growth abilities. (A) Expressions of $d n a E 1$ and dnaE2 in YL1605 (DK1622 att::dnaE2) and YL1604 (DK1622 att::Kan) in CTT growth medium; (B) Expressions of $d n a E 1$ and $d n a E 2$ in the two mutants on TPM plate; (C) Growth curves of YL1605 and YL1604 in CTT liquid medium supplemented with kanamycin $(40 \mu \mathrm{g} / \mathrm{ml})$. OD600 values were measured every $4 \mathrm{~h}$ until $60 \mathrm{~h}$ of incubation. The data were analyzed using two-way ANOVA. The error bars represent the standard deviation of three independent experiments. The expressions of dnaE1 and dnaE2 in (A) and (B) of this Figure were the relative expressions, compared with the expression of the dnaE1 gene in CTT at $12 \mathrm{~h}$ of nutritional condition of Figure 2A. panels), which is important for binding to Pol III $\tau$-subunit (Liu et al., 2013).

In order to get insight into the domain conservation, we estimated the $d N / d S$ values, an indicator of the selective pressure acting on a protein-coding gene, for the duplicated $d n a E$ genes of those sequenced myxobacteria. The $d N / d S$ values of the complete $d n a E 1$ and $d n a E 2$ sequences were 0.125 ( $95 \%$ confidence interval: $0.121-0.129$ ) and 0.172 (95\% confidence interval: $0.167-0.178$ ), respectively, suggesting both polymerases were subjected to a strong negative selection pressure for their strict functional constraint. The $d N / d S$ values were close between the Pol3, $\mathrm{HhH}$, and $\mathrm{OB}$ domain sequences of the dnaE1 and dnaE2 genes, but had a great difference in the PHP sequence $(t$ test, $P<0.01$, Figure 4B), which has a binding site of the proofreading $\varepsilon$-subunit (Wieczorek and McHenry, 2006; Ozawa et al., 2013). The $d N / d S$ value of the $d n a E 2$ PHP domain was more than twice than that of dnaE1. Accordingly, dnaE1 is more evolutionarily conservative than $d n a E 2$ in myxobacteria. Stano et al. reported that the PHP domain of DnaE-type polymerases had $3^{\prime}-5^{\prime}$ exonuclease activity (Stano et al., 2006). Similarly, the PHP domain of Mtb DnaE1 retains all amino acids associated with exonuclease activity in $M$. tuberculosis, but the DnaE2 PHP lost some key residues (Wieczorek and McHenry, 2006; Baños et al., 2008). It was speculated that the loss of conserved residues in the DnaE2 PHP domain affected relative fidelity. The DNA polymerase III complexes constructed with the two DnaE proteins thus probably had divergent functions.

\section{DISCUSSION}

DNA polymerase III holoenzyme is the main polymerase for bacterial DNA replication. While most bacterial cells contain single copies of $d n a E$, encoding for the alpha subunit of the polymerase, some bacteria contain duplicate $d n a E$ genes (Timinskas et al., 2014). There are several reports dealt with the functions of duplicated $d n a E$ genes and have revealed some key characteristics of $d n a E 2$, for example, the second $d n a E$ gene encodes an error-prone enzyme and is dispensable. However, the function of the duplicate dnaE is still an enigma. For example, what is the role of the second copy of $d n a E$ in bacteria, why do bacterial cells need an error-prone DNA polymerase enzyme, and how does the error-prone enzyme play functions in bacterial cells?

TABLE 3 | Sporulation abilities of M. xanthus YL1604 (DK1622 att::Kan) and YL1605 (DK1622 att:: dnaE2) cells grown on TPM plates treated with nalidixic acid for $1 \mathrm{~h}$ at different time points.

\begin{tabular}{|c|c|c|c|c|}
\hline Time of addition & Spore production in YL1604 & Percentage of control\% & Spore production in YL1605 & Percentage of control\% \\
\hline Control & $1.95 \times 10^{6} \pm 1.56 \times 10^{5}$ & 100 & $2.10 \times 10^{6} \pm 1.81 \times 10^{5}$ & 100 \\
\hline $\mathrm{Oh}$ & $1.26 \times 10^{5} \pm 8.62 \times 10^{3}$ & 6.43 & $1.93 \times 10^{5} \pm 8.74 \times 10^{3}$ & 9.21 \\
\hline $6 \mathrm{~h}$ & $1.27 \times 10^{6} \pm 1.21 \times 10^{5}$ & 65.02 & $1.83 \times 10^{6} \pm 1.56 \times 10^{5}$ & 86.98 \\
\hline $12 \mathrm{~h}$ & $1.88 \times 10^{6} \pm 1.32 \times 10^{5}$ & 96.25 & $2.09 \times 10^{6} \pm 1.85 \times 10^{5}$ & 99.37 \\
\hline $18 \mathrm{~h}$ & $2.00 \times 10^{6} \pm 1.71 \times 10^{5}$ & 102.56 & $2.13 \times 10^{6} \pm 1.50 \times 10^{5}$ & 101.27 \\
\hline $24 \mathrm{~h}$ & $2.08 \times 10^{6} \pm 1.69 \times 10^{5}$ & 108.89 & $2.21 \times 10^{6} \pm 2.01 \times 10^{5}$ & 105.4 \\
\hline
\end{tabular}




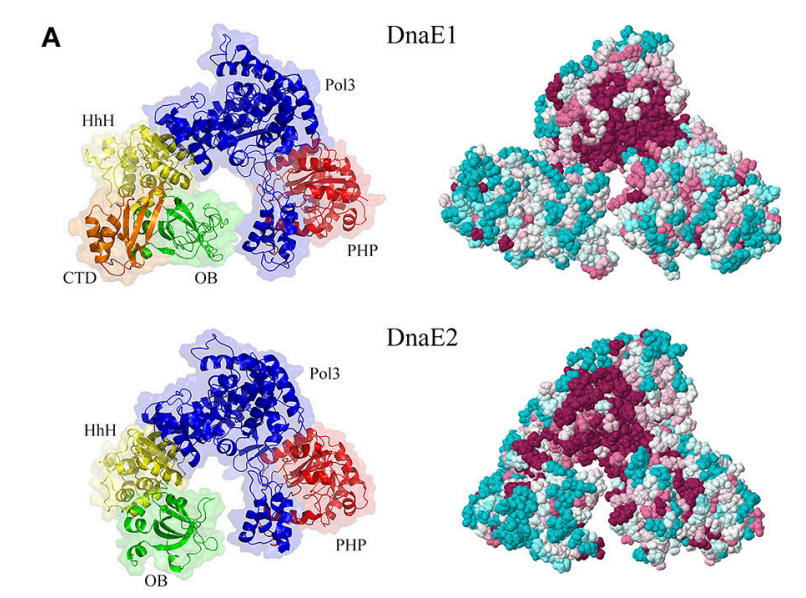

B

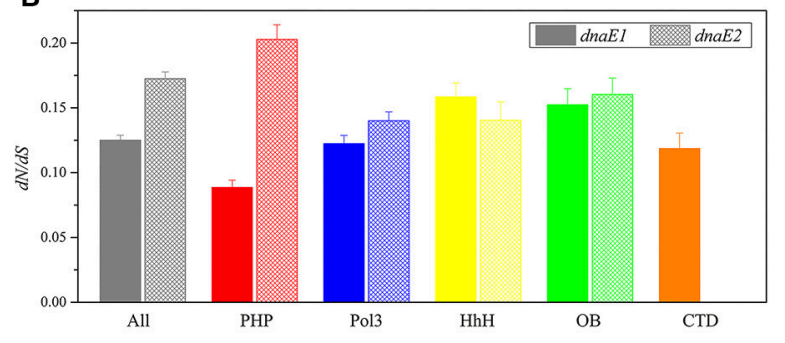

FIGURE 4 | Structure modeling of DK1622 DnaE1 and DnaE2 (A) and comparison of the domain conservation of myxobacterial dnaE1 and dnaE2 (B). (A) Three-dimensional structures showing different domains in different colors (left panels) and evolutionary conservation of surface amino acids (right panels); (B) The $d N / d S$ values of $d n a E 1$ and $d n a E 2$ and the different domains of those sequenced myxobacteria.

Myxobacteria have complex multicellular social behaviors, and all sequenced myxobacterial genomes contain duplicated dnaE genes. Given the high conservation in myxobacteria (Figure S1), the DnaE2 subunit should be important for myxobacterial cells. The expression of $d n a E 1$ was greatly higher than that of the dnaE2 gene throughout the lifecycle of $M$. xanthus cells. Obviously, the dnaE1 gene plays the major and essential role in chromosome replication in $M$. xanthus cells, not only in the growth stage but also in the development stage, thus ensuring the population genetically stable. In contrast, the $d n a E 2$ gene expressed approximately ten times lower than the $d n a E 1$ gene did in either the growth or the developmental stage, which is consistent with their weak cellular effects. Although weakly affected the growth and social motility, phenotypic characteristics of dnaE2 mutants supported that dnaE2 strongly affected the development progress. However, because the expression of $d n a E 1$ was also decreased, the deficiencies of development and sporulation in the dnaE2 deletion mutant were the results of the synergetic effects of the two dnaE genes.

In nutrition-limited conditions, $M$. xanthus populations aggregate and develop fruiting bodies, which contain stress resistant myxospores (Shimkets, 1990; Shimkets et al., 2006). Unlike the formation progress of endospores in Bacillus subtilis (Errington and Wake, 1991), M. xanthus cells carry out a round of chromosome replication in the early stage of development (Tzeng and Singer, 2005). We demonstrated that the deletion of dnaE2 caused obvious phenotypic deficiencies in development and sporulation. The development program of $M$. xanthus is a multicellular process requiring the coordinated expression of numerous regulation pathways to produce extracellular Ato E-signals and intracellular signals (Kaiser, 2004). Stringent response to the starvation of amino acids, the (p)ppGpp synthase enzyme (RelA) produces the chemical signal (p)ppGpp to initiate the development process (Singer and Kaiser, 1995; Harris et al., 1998). Rosario and Singer showed that inhibition of DNA replication within the first $6 \mathrm{~h}$ of development results in a block in developmental program, and the dnaA gene that encodes the initiator protein for DNA replication is not essential for development but allow for the proper timing and maximum efficiency of the sporulation process (Rosario and Singer, 2010). Our data improve the understanding of chromosome replication in the early stage of development of M. xanthus. We determined that, together with the essential DnaE1 polymerase, the error-prone DnaE2 enzyme participated in the chromosome replication of development process in M. xanthus DK1622, but in low fidelity for the genome replication due to its error-prone characteristic. It is known that suitable mutation rates can increase the adaptation abilities, whereas high mutation rates damage bacterial cells (Oliver et al., 2000; Giraud et al., 2001; Wielgoss et al., 2013). In new environments, the mutations probably increase for rapid adaptation, while in stable conditions, the mutations may decrease to reduce damages to cells. Thus, bacterial cells have to balance the mutation rates. The previous studies suggested that the bacterial land colonization is dominated by the emergence of $d n a E 2$, followed by a series of niche-specific genomic adaptations, including GC content increase, intensive horizontal gene transfer and constant genome expansion (Wu et al., 2014). We suggested that the DnaE2 is rather a balancer to control the genome mutations to a suitable rate, based on its error-prone characteristic and its low expressions in either the growth or development stage. Low concentrations make the replication carried out by the DnaE2 polymerases occupy small fractions. We found that artificial overexpression of the dnaE2 gene significantly increased the genome mutation rate, which suggested to be damaged for cell survivals. In addition, because the deletion of dnaE2 caused deficiency of development and sporulation, the presence of the gene had some more effects on cellular behaviors. Using the master strategy, $M$. xanthus populations are able to live through the changing environments.

\section{EXPERIMENTAL PROCEDURES}

\section{Strains and Cultivation}

The bacterial strains used in this study are listed in Table S2. The M. xanthus strains were cultivated in Casitone-based CTT medium (Hodgkin and Kaiser, 1977) for growth assays and on TPM agar plate (Kearns et al., 2000) for developmental assays. E. coli strains were routinely grown on Luria-Bertani (LB) agar or in LB liquid broth. M. xanthus strains were incubated at $30^{\circ} \mathrm{C}$, and 
E. coli strains were at $37^{\circ} \mathrm{C}$. When required, a final concentration of $40 \mu \mathrm{g} / \mathrm{ml}$ of kanamycin $(\mathrm{Km})$ was added to the solid or liquid media.

\section{Construction of $\boldsymbol{M}$. xanthus Mutants}

We performed the in-frame deletions of the dnaE1, dnaE2, mutS, and mutL genes in $M$. xanthus strains using pBJ113 plasmid, respectively. The plasmid contains a kanamycin resistant cassette for the first round of screening and a galK gene for the negative screening (Ueki et al., 1996). Briefly, homologous arms of dnaE1, dnaE2, mutS, or mutL were cloned with primers (listed in Table S3) and the products were inserted into the EcoRI/XbaI site of pBJ113. The resulting plasmid were introduced into $M$. xanthus DK1622 strains via electroporation $(1.25 \mathrm{kV}, 400 \mathrm{~W}$, $25 \mathrm{mF}, 0.1-\mathrm{cm}$ cuvette gap), respectively. The second round of screening was then performed on CTT plates containing $1 \%$ galactose (Sigma). The deletion mutants that grew on galactose but were sensitive to kanamycin were identified and verified by PCR amplification and sequencing. Restriction enzymes, DNA ligase, and other DNA enzymes were used according to the manufacturers' recommendations. All fragments were validated by Sanger 3730 sequencing.

\section{Complementation and Overexpression of dnaE2 Gene}

The dnaE2 gene and upstream 500 bp sequence that contains the whole promoter of $d n a E 2$ gene was cloned using the primer pair of MXAN_3982_NativeF and MXAN_3982_NativeR. The fragment and pSWU19 plasmid (replacing the tet resistant cassette of pSWU30 with a kanamycin resistant cassette) were digested with the $\mathrm{XbaI} / \mathrm{EcoRI}$ digestion solution. The two fragments were ligated with T4 DNA ligase, producing the recombinant plasmid pSW3982. The promoter of the pilA gene was cloned using the primer pair of MXAN_3982P_UF and MXAN_3982P_UR, and fused with the dnaE2 gene. The fusion product was inserted into XbaI/EcoRI sites of pSWU19, producing the plasmid pSWp3982.

The dnaE2 fusion construct was transferred by electroporation into M. xanthus DK1622. Individual kanamycinresistant clones were selected, and the mutants of the dnaE2 transcription fusion were verified by PCR amplification (primers are listed in Table S3) and sequencing.

\section{Mutation Rate Assay}

The mutation rate assay was conducted by screening the nalidixic acid resistant strains according to previous report (Tzeng et al., 2006). Briefly, approximately $5 \times 10^{9}$ cells of $M$. xanthus strains were placed on CTT agar containing $40 \mu \mathrm{g} / \mathrm{ml}$ nalidixic acid. Nalidixic acid- resistant candidates were counted and mutation rate was calculated using the following formula, $r=N_{m} / N_{0}$, where $r$ is the mutation rate, $N_{m}$ represents the count of nalidixic acid resistant mutant, and $N_{0}$ is the count of cells placed on the screening plate.

\section{Development and Sporulation Assays}

Developmental experiments were performed on TPM agar plates (10 mM Tris [pH 7.6], $8 \mathrm{mM} \mathrm{MgSO}_{4}$, and $1 \mathrm{mM} \mathrm{KH}_{2} \mathrm{PO}_{4}$ containing $1.5 \%$ agar), as described below. Cells were allowed to develop in a humidity chamber at $30^{\circ} \mathrm{C}$. When indicated, nalidixic acid solution of the $20 \mu \mathrm{g} / \mathrm{ml}$ concentration was added onto different plates at the time of $0,6,12,18$, or $24 \mathrm{~h}$ for $1 \mathrm{~h}$, and then the extra solution was poured out. After $120 \mathrm{~h}$ cultivation at $30^{\circ} \mathrm{C}$. Sporulation was conducted as follows: five colonies were collected and resuspended in liquid CTT media. Sonication was conducted at $200 \mathrm{~W}$ for $4 \mathrm{~s}$ twice to release myxospores from fruiting bodies. The myxospore suspensions were incubated at $55^{\circ} \mathrm{C}$ for $2 \mathrm{~h}$ to kill the vegetative cells. Then the suspensions were serially diluted and aliquots of $50 \mu \mathrm{L}$ were used to plate. The sporulation rate was counted after 5 days of cultivation on the CTT plate.

\section{RNA Extraction and Quantitative Real-Time PCR Assay}

The $M$. xanthus DK1622 cells were concentrated to approximately $1.75 \times 10^{10}$ cells per milliliter. The cell suspension was washed three times using TPM buffer. RNA was extracted immediately with a BIOZOL total RNA extraction reagent (BioFlux) following the manufacturer's instructions. Genomic DNA contamination was removed by using DNA eraser supplied in the PrimeScript RT Reagent kit with gDNA Eraser (TaKaRa). The purified RNA extracts were transcribed reversely to cDNA and stored in aliquots at $-80^{\circ} \mathrm{C}$. Quantitative real-time PCR was performed in a total reaction volume of $25 \mu \mathrm{l}$, containing $250 \mathrm{nM}$ primers, $12.5 \mu \mathrm{l}$ of SYBR Premix Ex Taq GC mix (TaKaRa), $10.5 \mu \mathrm{l}$ of RNase-free water (TaKaRa), and $1 \mu \mathrm{l}$ of a 10-fold-diluted cDNA template. PCR was conducted in a Roche LightCycler 480 sequence detection system, following the program: $3 \mathrm{~min}$ at $95^{\circ} \mathrm{C}$, followed by 40 cycles of $30 \mathrm{~s}$ at $95^{\circ} \mathrm{C}, 30 \mathrm{~s}$ at $55^{\circ} \mathrm{C}$, and $15 \mathrm{~s}$ at $72^{\circ} \mathrm{C}$. The gapA gene, encoding for glyceraldehyde-3-phosphate dehydrogenase, was used as the normalization signal. Calibration curves of gapA, MXAN_3982, and MXAN_5844 were generated from a 10-fold dilution of $M$. xanthus DK1622 genomic DNA. The primer pairs used for each gene are listed in Table S3.

\section{Bioinformatics Analyses}

The dnaE1 and dnaE2 genes were extracted from the genome of $M$. xanthus DK1622 strain. All the dnaE genes used for phylogenetic analysis were extracted according to the Genomic Encyclopedia of Bacteria and Archaea (Wu et al., 2009). These genes were translated into amino acid sequences according to the bacterial standard codon. A multiple sequence alignment of the DnaE1 and DnaE2 proteins was established using the MAFFT program (Katoh and Standley, 2013). The phylogenetic tree was constructed using the PhyML program with the LG substitution model and the four substitution rate categories (Guindon et al., 2010). LG model was regarded as the best substitution model by automatic model selection. Branch support was calculated using the approximate likelihood ratio tests (aLRT SH-like) (Anisimova and Gascuel, 2006). The phylogenetic tree was visualized by iTOL (Letunic and Bork, 2016).

The structures of the DnaE1 and DnaE2 were modeled using the I-TASSER program based on a threading approach 
(Yang et al., 2015). The evolutionary conservation of amino acid positions in the $d n a E 1$ and $d n a E 2$ sequences was estimated by using the ConSurf algorithm (Ashkenazy et al., 2010). The JTT substitution matrix was used and the computation was based on the empirical Bayesian paradigm. The conservation scale was defined from the most variable amino acid positions (grade 1), which were considered to be evolved rapidly, to the most conservative positions (grade 9), which were considered to be evolved slowly. The sequences and modeled structures of the DnaE1 and DnaE2 were shown in nine-color conservation grades.

The number ratio of nonsynonymous substitutions per nonsynonymous site $(d N)$ to synonymous substitutions per synonymous site $(d S), d N / d S$, is an indicator of the selective pressure acting on a protein-coding gene. The $d N / d S$ calculation of the dnaE1 and dnaE2 locus was set at the 95\% confidence intervals, using the Datamonkey web server (Delport et al., 2010). For the calculation, a multiple sequence alignment of the nucleic acid sequences of myxobacterial $d n a E 1$ and $d n a E 2$ genes, based on codon, was established with TranslatorX (Abascal et al., 2010). Domains of DnaE1 and DnaE2 proteins were extracted by blasting against the PFAM database (Finn et al., 2014).

The statistical analysis was conducted using IBM SPSS Statistics.

\section{REFERENCES}

Abascal, F., Zardoya, R., and Telford, M. J. (2010). TranslatorX: multiple alignment of nucleotide sequences guided by amino acid translations. Nucleic Acids Res 38, W7-W13. doi: 10.1093/nar/gkq291

Abella, M., Erill, I., Jara, M., Mazón, G., Campoy, S., and Barbé, J. (2004). Widespread distribution of a lexA-regulated DNA damage-inducible multiple gene cassette in the Proteobacteria phylum. Mol. Microbiol. 54, 212-222. doi: 10.1111/j.1365-2958.2004.04260.x

Anisimova, M., and Gascuel, O. (2006). Approximate likelihood-ratio test for branches: a fast, accurate, and powerful alternative. Syst. Biol. 55, 539-552. doi: 10.1080/10635150600755453

Ashkenazy, H., Erez, E., Martz, E., Pupko, T., and Ben-Tal, N. (2010). ConSurf 2010: calculating evolutionary conservation in sequence and structure of proteins and nucleic acids. Nucleic Acids Res. 38, W529-W533. doi: 10.1093/nar/gkq399

Baños, B., Lázaro, J. M., Villar, L., Salas, M., and De Vega, M. (2008). Editing of misaligned 3'-termini by an intrinsic 3'-5' exonuclease activity residing in the PHP domain of a family X DNA polymerase. Nucleic Acids Res. 36, 5736-5749. doi: $10.1093 /$ nar/gkn526

Boshoff, H. I., Reed, M. B., Barry, C. E. III, and Mizrahi, V. (2003). DnaE2 polymerase contributes to in vivo survival and the emergence of drug resistance in Mycobacterium tuberculosis. Cell 113, 183-193. doi: 10.1016/S0092-8674(03)00270-8

Brinkhoff, T., Fischer, D., Vollmers, J., Voget, S., Beardsley, C., Thole, S., et al. (2012). Biogeography and phylogenetic diversity of a cluster of exclusively marine myxobacteria. ISME J. 6, 1260-1272. doi: 10.1038/ismej.2011.190

Delport, W., Poon, A. F., Frost, S. D., and Kosakovsky Pond, S. L. (2010). Datamonkey 2010: a suite of phylogenetic analysis tools for evolutionary biology. Bioinformatics 26, 2455-2457. doi: 10.1093/bioinformatics/btq429

Dworkin, M. (1996). Recent advances in the social and developmental biology of the myxobacteria. Microbiol. Rev. 60, 70-102.

Errington, J., and Wake, R. G. (1991). Chromosome strand segregation during sporulation in Bacillus subtilis. Mol. Microbiol. 5, 1145-1149. doi: 10.1111/j.1365-2958.1991.tb01887.x

\section{AUTHOR CONTRIBUTIONS}

Conceived and designed the experiments: YL and RP. Performed the experiments and analyzed the data: RP, JC, WF, ZZ, JY, and ZL. Wrote the paper: YL and RP.

\section{ACKNOWLEDGMENTS}

We thank Dr. K. Han for invaluable help with the manuscript. This work was financially supported by the National Natural Science Foundation of China (NSFC) (No. 31471183) and the NSFC Key Program (No. 31130004) awarded to YL.

\section{SUPPLEMENTARY MATERIAL}

The Supplementary Material for this article can be found online at: http://journal.frontiersin.org/article/10.3389/fmicb. 2017.00122/full\#supplementary-material

Figure S1 | Phylogenetic analyses of myxobacterial DnaE protein sequences. Some determined DnaE1 and DnaE2 proteins from other bacteria species were also included in the tree.

Table S1 | Information of dnaE genes in sequenced myxobacterial genomes.

Table S2 | Strains and plasmids used in this study.

Table S3 | Primers used in this study.

Evans, R. J., Davies, D. R., Bullard, J. M., Christensen, J., Green, L. S., Guiles, J. W., et al. (2008). Structure of PolC reveals unique DNA binding and fidelity determinants. Proc. Natl. Acad. Sci. U.S.A. 105, 20695-20700. doi: 10.1073/pnas.0809989106

Finn, R. D., Bateman, A., Clements, J., Coggill, P., Eberhardt, R. Y., Eddy, S. R., et al. (2014). Pfam: the protein families database. Nucleic Acids Res. 42, D222-D230. doi: $10.1093 /$ nar/gkt1223

Giraud, A., Matic, I., Tenaillon, O., Clara, A., Radman, M., Fons, M., et al. (2001). Costs and benefits of high mutation rates: adaptive evolution of bacteria in the mouse gut. Science 291, 2606-2608. doi: 10.1126/science.10 56421

Goldman, B. S., Nierman, W. C., Kaiser, D., Slater, S. C., Durkin, A. S., Eisen, J. A., et al. (2006). Evolution of sensory complexity recorded in a myxobacterial genome. Proc. Natl. Acad. Sci. U.S.A. 103, 15200-15205. doi: 10.1073/pnas.0607335103

Guindon, S., Dufayard, J. F., Lefort, V., Anisimova, M., Hordijk, W., and Gascuel, O. (2010). New algorithms and methods to estimate maximum-likelihood phylogenies: assessing the performance of PhyML 3.0. Syst. Biol. 59, 307-321. doi: $10.1093 /$ sysbio/syq010

Harris, B. Z., Kaiser, D., and Singer, M. (1998). The guanosine nucleotide (p)ppGpp initiates development and A-factor production in Myxococcus xanthus. Genes Dev. 12, 1022-1035. doi: 10.1101/gad.12.7.1022

Hodgkin, J., and Kaiser, D. (1977). Cell-to-cell stimulation of movement in nonmotile mutants of Myxococcus. Proc. Natl. Acad. Sci. U.S.A. 74, 2938-2942. doi: 10.1073/pnas.74.7.2938

Ito, J., and Braithwaite, D. K. (1991). Compilation and alignment of DNA polymerase sequences. Nucleic Acids Res. 19, 4045-4057. doi: 10.1093/nar/19.15.4045

Jiang, D. M., Kato, C., Zhou, X. W., Wu, Z. H., Sato, T., and Li, Y. Z. (2010). Phylogeographic separation of marine and soil myxobacteria at high levels of classification. ISME J. 4, 1520-1530. doi: 10.1038/ismej.2010.84

Kaiser, D. (2004). Signaling in myxobacteria. Annu. Rev. Microbiol. 58, 75-98. doi: 10.1146/annurev.micro.58.030603.123620

Kaiser, D., and Losick, R. (1993). How and why bacteria talk to each other. Cell 73, 873-885. doi: 10.1016/0092-8674(93)90268-U 
Katoh, K., and Standley, D. M. (2013). MAFFT multiple sequence alignment software version 7: improvements in performance and usability. Mol. Biol. Evol. 30, 772-780. doi: 10.1093/molbev/mst010

Kearns, D. B., Campbell, B. D., and Shimkets, L. J. (2000). Myxococcus xanthus fibril appendages are essential for excitation by a phospholipid attractant. Proc. Natl. Acad. Sci. U.S.A. 97, 11505-11510. doi: 10.1073/pnas.210448597

Kelman, Z., and O'donnell, M. (1995). DNA polymerase III holoenzyme: structure and function of a chromosomal replicating machine. Annu. Rev. Biochem. 64, 171-200. doi: 10.1146/annurev.bi.64.070195.001131

Koorits, L., Tegova, R., Tark, M., Tarassova, K., Tover, A., and Kivisaar, M. (2007). Study of involvement of ImuB and DnaE2 in stationary-phase mutagenesis in Pseudomonas putida. DNA Repair (Amst). 6, 863-868. doi: 10.1016/j.dnarep.2007.01.010

Lamers, M. H., Georgescu, R. E., Lee, S. G., O'donnell, M., and Kuriyan, J. (2006). Crystal structure of the catalytic $\alpha$ subunit of $E$. coli replicative DNA polymerase III. Cell 126, 881-892. doi: 10.1016/j.cell.2006.07.028

Letunic, I., and Bork, P. (2016). Interactive tree of life (iTOL) v3: an online tool for the display and annotation of phylogenetic and other trees. Nucleic Acids Res. 44, W242-W245. doi: 10.1093/nar/gkw290

Li, S. G., Zhou, X. W., Li, P. F., Han, K., Li, W., Li, Z. F., et al. (2012). The existence and diversity of myxobacteria in lake mud - a previously unexplored myxobacteria habitat. Environ. Microbiol. Rep. 4, 587-595. doi: 10.1111/j.1758-2229.2012.00373.x

Liu, B., Lin, J., and Steitz, T. A. (2013). Structure of the PolIII $\alpha$-tauc-DNA complex suggests an atomic model of the replisome. Structure 21, 658-664. doi: 10.1016/j.str.2013.02.002

McHenry, C. S. (2011a). Bacterial replicases and related polymerases. Curr. Opin. Chem. Biol. 15, 587-594. doi: 10.1016/j.cbpa.2011.07.018

McHenry, C. S. (2011b). Breaking the rules: bacteria that use several DNA polymerase IIIs. EMBO Rep. 12, 408-414. doi: 10.1038/embor.2011.51

Oliver, A., Cantón, R., Campo, P., Baquero, F., and Blázquez, J. (2000). High frequency of hypermutable Pseudomonas aeruginosa in cystic fibrosis lung infection. Science 288, 1251-1254. doi: 10.1126/science.288.546 9.1251

Ozawa, K., Horan, N. P., Robinson, A., Yagi, H., Hill, F. R., Jergic, S., et al. (2013). Proofreading exonuclease on a tether: the complex between the $E$. coli DNA polymerase III subunits $\alpha, \varepsilon, \theta$, and $\beta$ reveals a highly flexible arrangement of the proofreading domain. Nucleic Acids Res. 41, 5354-5367. doi: $10.1093 / \mathrm{nar} / \mathrm{gkt} 162$

Reichenbach, H. (1999). The ecology of the myxobacteria. Environ. Microbiol. 1, 15-21. doi: 10.1046/j.1462-2920.1999.00016.x

Robinson, A., Causer, R. J., and Dixon, N. E. (2012). Architecture and conservation of the bacterial DNA replication machinery, an underexploited drug target. Curr. Drug Targets 13, 352-372. doi: 10.2174/138945012799424598

Rosario, C. J., and Singer, M. (2010). Developmental expression of dnaA is required for sporulation and timing of fruiting body formation in Myxococcus xanthus. Mol. Microbiol. 76, 1322-1333. doi: 10.1111/j.1365-2958.2010.07178.x

Shimkets, L. J. (1990). Social and developmental biology of the myxobacteria. Microbiol. Rev. 54, 473-501.

Shimkets, L. J., Dworkin, M., and Reichenbach, H. (2006). "The myxobacteria," in The prokaryotes, eds M. Dworkin, S. Falkow, E. Rosenberg, K. H. Schleifer, and E. Stackebrandt (New York, NY: Springer), 31-115.

Singer, M., and Kaiser, D. (1995). Ectopic production of guanosine penta- and tetraphosphate can initiate early developmental gene expression in Myxococcus xanthus. Genes Dev. 9, 1633-1644. doi: 10.1101/gad.9.13.1633

Sniegowski, P. D., Gerrish, P. J., and Lenski, R. E. (1997). Evolution of high mutation rates in experimental populations of E. coli. Nature 387, 703-705. doi: $10.1038 / 42701$
Stano, N. M., Chen, J., and McHenry, C. S. (2006). A coproofreading Zn $2^{+}$dependent exonuclease within a bacterial replicase. Nat. Struct. Mol. Biol. 13, 458-459. doi: 10.1038/nsmb1078

Timinskas, K., Balvociute, M., Timinskas, A., and Venclovas, C. (2014). Comprehensive analysis of DNA polymerase III $\alpha$ subunits and their homologs in bacterial genomes. Nucleic Acids Res. 42, 1393-1413. doi: 10.1093/nar/gkt900

Tsai, H. H., Shu, H. W., Yang, C. C., and Chen, C. W. (2012). Translesion-synthesis DNA polymerases participate in replication of the telomeres in Streptomyces. Nucleic Acids Res. 40, 1118-1130. doi: 10.1093/nar/gkr856

Tzeng, L., Ellis, T. N., and Singer, M. (2006). DNA replication during aggregation phase is essential for Myxococcus xanthus development. J. Bacteriol. 188, 2774-2779. doi: 10.1128/JB.188.8.2774-2779.2006

Tzeng, L., and Singer, M. (2005). DNA replication during sporulation in Myxococcus xanthus fruiting bodies. Proc. Natl. Acad. Sci. U.S.A. 102, 14428-14433. doi: 10.1073/pnas.0506969102

Ueki, T., Inouye, S., and Inouye, M. (1996). Positive-negative KG cassettes for construction of multi-gene deletions using a single drug marker. Gene 183, 153-157. doi: 10.1016/S0378-1119(96)00546-X

Warner, D. F., Ndwandwe, D. E., Abrahams, G. L., Kana, B. D., Machowski, E. E., Venclovas, C., et al. (2010). Essential roles for imuA'- and imuB-encoded accessory factors in DnaE2-dependent mutagenesis in Mycobacterium tuberculosis. Proc. Natl. Acad. Sci. U.S.A. 107, 13093-13098. doi: $10.1073 /$ pnas. 1002614107

Wieczorek, A., and McHenry, C. S. (2006). The NH2-terminal php domain of the $\alpha$ subunit of the Escherichia coli replicase binds the epsilon proofreading subunit. J. Biol. Chem. 281, 12561-12567. doi: 10.1074/jbc.M513844200

Wielgoss, S., Barrick, J. E., Tenaillon, O., Wiser, M. J., Dittmar, W. J., Cruveiller, S., et al. (2013). Mutation rate dynamics in a bacterial population reflect tension between adaptation and genetic load. Proc. Natl. Acad. Sci. U.S.A. 110, 222-227. doi: 10.1073/pnas.1219574110

Wu, D., Hugenholtz, P., Mavromatis, K., Pukall, R., Dalin, E., Ivanova, N. N., et al. (2009). A phylogeny-driven genomic encyclopaedia of Bacteria and Archaea. Nature 462, 1056-1060. doi: 10.1038/nature08656

Wu, H., Fang, Y., Yu, J., and Zhang, Z. (2014). The quest for a unified view of bacterial land colonization. ISME J. 8, 1358-1369. doi: 10.1038/ismej.2013.247

Wu, S. S., and Kaiser, D. (1995). Genetic and functional evidence that Type IV pili are required for social gliding motility in Myxococcus xanthus. Mol. Microbiol. 18, 547-558. doi: 10.1111/j.1365-2958.1995.mmi_18030547.x

Yang, J., Yan, R., Roy, A., Xu, D., Poisson, J., and Zhang, Y. (2015). The ITASSER Suite: protein structure and function prediction. Nat. Methods 12, 7-8. doi: $10.1038 /$ nmeth.3213

Zhao, X., Zhang, Z., Yan, J., and Yu, J. (2007). GC content variability of eubacteria is governed by the pol III $\alpha$ subunit. Biochem. Biophys. Res. Commun. 356, 20-25. doi: 10.1016/j.bbrc.2007.02.109

Zhou, X. W., Li, S. G., Li, W., Jiang, D. M., Han, K., Wu, Z. H., et al. (2014). Myxobacterial community is a predominant and highly diverse bacterial group in soil niches. Environ. Microbiol. Rep. 6, 45-56. doi: 10.1111/1758-2229.12107

Conflict of Interest Statement: The authors declare that the research was conducted in the absence of any commercial or financial relationships that could be construed as a potential conflict of interest.

Copyright (c) 2017 Peng, Chen, Feng, Zhang, Yin, Li and Li. This is an open-access article distributed under the terms of the Creative Commons Attribution License (CC $B Y)$. The use, distribution or reproduction in other forums is permitted, provided the original author(s) or licensor are credited and that the original publication in this journal is cited, in accordance with accepted academic practice. No use, distribution or reproduction is permitted which does not comply with these terms. 